${ }^{1}$ Departamento

de Dermatología,

Pontificia Universidad

Católica de Chile,

Santiago, Chile.

${ }^{2}$ Interna de medicina,

Escuela de Medicina,

Pontificia Universidad

Católica de Chile,

Santiago, Chile.

Trabajo no recibió financiamiento.

Los autores declaran no tener conflictos de interés.

Recibido el 23 de octubre de 2019 , aceptado el 10 de abril de 2020.

Correspondencia a: María José Zambrano Mericq. Email:mjzambra@ uc.cl

Artículo de Investigación

\title{
Significativo Aumento de Onicomicosis por Hongos Filamentosos No Dermatofitos en Red de Salud Privada en Santiago de Ghile entre los períodos 2008-2009 y 2016-2017
}

Celeste Martin ${ }^{1}$, María José Zambrano ${ }^{1}$, Natalia Sabatini ${ }^{1}$, Suelí Muñoz ${ }^{2}$, Vivian Tabak ${ }^{2}$, Marcela Concha ${ }^{1}$, Félix Fich ${ }^{1}$

\section{RESUMEN}

Introducción: La onicomicosis corresponde a una patología prevalente causada por hongos dermatofitos, levaduras y en menor proporción, hongos filamentosos no dermatofitos (HFND). Se ha reportado un aumento a nivel mundial en la incidencia de onicomicosis por $\mathrm{HFND}^{1}$, sin embargo, en Chile no hay registros de ello.

Objetivo: Comparar la epidemiología local de onicomicosis por HFND en una red privada de Santiago de Chile entre dos períodos de tiempo.

Material y métodos: Se realizó un estudio transversal de análisis de los registros de laboratorio de la Red de Salud UC-Christus entre los períodos 20082009 y 2016-2017.

Resultados: Se obtuvieron un total de 9.579 muestras en ambos períodos, 4.985 entre 2008-2009 y 4.594 entre $2016-2017$, con $3.442(36 \%)$ cultivos positivos para hongos, $1.831(36,7 \%)$ en el primer período y $1.611(35 \%)$ en el segundo. Del total 40 muestras $(1,2 \%)$ fueron de HFND, destacando que la prevalencia aumentó significativamente entre ambos períodos: $8(0,4 \%)$ y $32(2,0 \%)$ cultivos, respectivamente $(\mathrm{p}<0,0001)$. Del total, las especies de HFND más prevalentes fueron: 23 Fusarium sp $(57,5 \%), 8$ Scopulariopsis sp $(20 \%)$ y 7 Acremonium sp $(17,5 \%)$.

En pacientes con cultivo positivo para HFND, se buscaron asociaciones probables en los antecedentes a través de la ficha clínica, sin encontrar resultados significativos.

Conclusión: Los HFND son la tercera causa de onicomicosis y su prevalencia fue 1,2\%. Las especies más frecuentes fueron Fusarium sp, Scopulariopsis sp, Acremonium sp. La prevalencia de HFND aumentó significativamente en un período de 6 años.

Palabras claves: Onicomicosis; dermatofitos no filamentosos; Fusarium; epidemiología.

\section{SUMMARY}

Introduction: Onychomycosis is a frequent nail disease caused mainly by dermatophytes, in less proportion yeast and last non-dermatophyte molds (NDM). NDM onychomycosis is an increasing problem worldwide, though in Chile there is no epidemiological registry about it.

Objetive: The aim of the study was to determine the local epidemiology of NDM onychomycosis.

Materials y methods: We did a transversal cohort study in the Red de Salud UC-Christus between 2008-2009 and 2016-2017.

Results: Of the 9,579 clinically suspected cases of onychomycosis, 4,985 in 2008-2009 and 4,594 in 2016-2017, 3,448 (36\%) cultures were positive in total, 1,831 $(36.7 \%)$ in the first period and 1,611 $(35 \%)$ in the second. Only 40 cultures $(1.2 \%)$ were NDM in total, but the prevalence significantly increased between both periods with $8(0.45 \%)$ and $32(2.0 \%)$ cultures, respectively $(\mathrm{p}<0.0001)$. Among the total of NDM, the most prevalent species were: 23 Fusarium sp $(57.5 \%)$, 8 Scopulariopsis sp $(20 \%)$ and 7 Acremonium sp $(17.5 \%)$.

We searched for possible associations of patients with NDM onychomycosis and morbid history, but found no significant result.

Conclusion: NDM are the third cause of onychomycosis and its prevalence of $1.2 \%$ was predominantly from toe nails. The most frequent species were Fusarium sp, Scopulariopsis sp. and Acremonium sp. The prevalence of NDM increased significantly in a period of 6 years.

Key words: Onychomycosis; mitosporic fungi; nondermatophyte molds; Fusarium. 
$\mathrm{L}$ a onicomicosis corresponde a una patología frecuente en la práctica dermatológica, estimada entre el 50 al $60 \%$ de todas las consultas por alteraciones ungueales. ${ }^{2}$ La prevalencia de onicomicosis varía en los distintos estudios poblacionales, con estimaciones que van desde el 8 al 27\%. ${ }^{2-4}$ Se presenta con mayor frecuencia en los adultos y en individuos de mayor edad.

Los agentes fúngicos involucrados son: 1) dermatofitos -que corresponde al grupo más frecuente, 2) levaduras -que frecuentemente se asocian a inmunosupresión o enfermedades endocrinológicas, como la diabetes mellitus, y 3) hongos filamentosos no dermatofitos o mohos (HFND). ${ }^{5}$ Clínicamente, el agente causal es indistinguible.

Los HFND son frecuentes en zonas tropicales y templadas. Incluyen más de 60 especies, 10 de las cuales son patógenos humanos conocidos, pero las más frecuentes identificadas como causantes de onicomicosis son: Scopulariopsis brevicaulis, Aspergillus sp, Fusarium sp, Acremonium sp, Scytalidium sp, Onychocola canadiensis y Arachnomyces kanei. ${ }^{6}$ Los géneros Aspergillus y Fusarium se encuentran entre los más prevalentes causantes de onicomicosis a nivel mundial. Se ha descrito que las especies del género Fusarium pueden causar una enfermedad localizada en individuos inmunocompetentes e incluso diseminada en pacientes inmunosuprimidos. ${ }^{7}$

Se ha discutido por muchos años

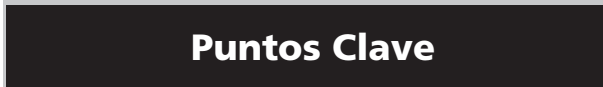

- La onicomicosis es una consulta dermatológica común. Los hongos filamentosos no dermatofitos son un agente causal infrecuente. Hay pocos estudios epidemiológicos en Chile.

- Esta investigación muestra que en los últimos años ha habido un aumento significativo de onicomicosis por hongos no dermatofitos.

- Actualizar a nuestro medio para que tengamos una mayor sospecha diagnóstica de onicomicosis por agentes filamentosos no dermatofitos y así realizar un tratamiento adecuado. si los HFND pueden ser causantes

de onicomicosis, pues en su mayoría son saprófitos que se encuentran en piel sana, en la tierra, plantas, otros sustratos orgánicos y en el ambiente de laboratorios. Es por ello que el diagnóstico se debe basar en criterios estrictos, descartando que sea un comensal o un contaminante ambiental. Se ha descrito que crecen en uñas previamente traumatizadas, desvitalizadas por patologías circulatorias o en pacientes inmunosuprimidos; sin embargo, en otros casos afectan a personas sanas y con uñas sanas. ${ }^{6}$
Con respecto al mecanismo de patogenicidad de los HFND, se ha demostrado que Fusarium es capaz de cretruir el estrato córneo, sugiriendo que es queratinolítico Una dificultad en el diagnóstico específico es la identificación del patógeno, la cual se basa principalmente en sus características morfológicas, a menudo muy sutiles entre ellos.

Actualmente se reconoce que los HFND son patógenos e onicomicosis y según los últimos reportes en la literaura, su prevalencia es muy variada y ha ido en aumento: ,89\% en Venezuela, ${ }^{6} 12,4 \%$ en Ciolombia, ${ }^{10} 17,2 \%$ España, ${ }^{11} 22 \%$ en India $^{12}$ y $51,6 \%$ en Tailandia. ${ }^{13}$ En Chile existen escasos estudios poblacionales, el más reciente realizado en la región de Valparaíso el año 2011 , en que $4,6 \%$ de las onicomicosis fueron por HFND, y el principal agente fue Fusarium $(55,5 \%) .^{14}$ La prevalencia de Fusarium varía entre 1 y $16 \%$, con ejemplos como Brasil con 12,4\%, Estados Unidos con $6,9 \%$ y Colombia con $6 \% .^{15}$

La prevalencia de infecciones diseminadas por HFND ha aumentado, afectando principalmente a pacientes inmunocomprometidos. ${ }^{16}$ La infección puede adquirirse por inhalación de conidias, con posterior diseminación hematógena, o vía cutánea, principalmente secundario a onicomicosis. En una unidad de hematología en EEUU, 66,7\% de las fusariosis invasoras tenían como puerta de entrada la piel, en su mayoría onicomicosis y/o tiña interdigital de los pies. ${ }^{17}$ Por ello es fundamental el diagnóstico adecuado y tratamiento precoz, ya que esto puede prevenir una fusariosis invasora en pacientes inmunosuprimidos, lo cual es una infección con alta morbi-mortalidad. Además, muchos HFND presentan resistencia a los antifúngicos clásicos, por lo que se requieren tratamientos más prolongados, combinación de terapia sistémica y tópica y/o uso de otros agentes, como itraconazol. ${ }^{1}$ 
El presente estudio pretende determinar la prevalencia de onicomicosis causadas por HFND en la Red Salud UC-Christus, objetivar si existe un aumento de su prevalencia en los períodos 2008-2009 y 2016-2017 y su relación con variables sociodemográficas, clínicas y patológicas de los pacientes.

\section{Materiales y Métodos}

Se realizó un estudio transversal en la Red de Salud Christus, la cual es una red de atención médica privada que atiende a pacientes de diferentes estratos socioeconómicos y sectores geográficos de la ciudad de Santiago. Se recopilaron datos del registro manuscrito de los micológicos directos y cultivos de hongos del laboratorio de microbiología de la Red de Salud UC-Christus entre enero y diciembre de los años 2008, 2009, 2016 y 2017.

En el laboratorio clínico de la Red de Salud UC-Christus las muestras se obtuvieron mediante raspado de la superficie de la uña, la cual es tratada con hidróxido de potasio al $20 \%$ y tinta china, posterior a lo cual se visualizaron al microscopio (micológico directo) y luego fueron cultivadas a $30^{\circ} \mathrm{C}$ en 2 medios: agar dextrosa Sabouraud con cloranfenicol y en agar lactrimel de Borelli con la adición de cloranfenicol y cicloheximida. Los tubos fueron analizados periódicamente por 30 días. Se visualizaron las colonias macroscópicamente y luego microscópicamente para identificar la especie causante. Se consideraron positivos los cultivos para HFND cuando en ambos tubos sembrados en medio Saboraud y Borelli creció una colonia de HFND y hubo ausencia de otra especie de hongo (levadura o dermatofitos). Esta técnica es estandarizada, y en ambos períodos de tiempo se mantuvieron los mismos operadores, técnica, medios de cultivo y el lugar de trabajo.

En los pacientes cuyo cultivo resultó positivo para HFND, se consultó en la ficha clínica sobre antecedentes mórbidos, uso de medicamentos habituales, medicamentos en los últimos 6 meses (antibióticos, terapia antimicótica, inmunosupresores, entre otros) y tratamientos utilizados para onicomicosis.

Se realizó análisis estadístico descriptivo y las variables categóricas fueron evaluadas mediante test chi-cuadrado, con valor $\mathrm{p}$ significativo $<0,05$. El IC del 95\% se proporcionó cuando fue adecuado.

\section{Resultados}

Se analizaron en total 9.579 muestras de micológico directo y cultivo de hongos realizados en los años 2008, 2009, 2016 y 2017 del laboratorio de microbiología de la Red de Salud UG-Christus, de las cuales 4.985 muestras fueron obtenidas entre 2008-2009 y 4.594 entre 20162017. Del total de cultivos, $41 \%$ corresponden a sexo masculino ( $42 \%$ en el primer período y $40 \%$ en el segundo), $59 \%$ a sexo femenino (58\% en el primer período, $60 \%$ en el segundo) y la edad promedio fue $44,6 \pm 19,3$ años (rango 2-108 años), similares en ambos períodos (44 $\pm 19,2$ en el primero y 45,3 $\pm 19,5$ en el segundo). Del total de cultivos, $86 \%$ fueron muestras de uñas de pies (85\% entre $2008-2009$ y $87 \%$ entre $2016-2017)$ y $14 \%$ de uñas de manos (15\% en el primer período y $13 \%$ en el segundo).

El total de cultivos positivos para hongos fue 3.442 (36\% del total de cultivos), correspondiendo a $1.831(36,7 \%)$ en el primer período y 1.611 (35\%) en el segundo período. De estos cultivos, fueron dermatofitos $1.473(80,4 \%)$ en el primer período y $1.398(86,8 \%)$ en el segundo, con un total de $2.871(83,4 \%)$. El resultado de cándida fue 350 $(19,1 \%)$ en el primer período, $181(11,2 \%)$ en el segundo y en total $531(15.4 \%)$. Con respecto a los HFND, el total fue $40(1,2 \%)$ HFND, obteniendo $8(0,4 \%)$ en el primer período y $32(2,0 \%)$ en el segundo. Además, se pesquisaron en total 6 cultivos $(0.1 \%)$ del género Pseudomonas, 1 en el primer período y 5 en el segundo (Tabla 1).

Del total de cultivos, con respecto a los dermatofitos, el más frecuente fue Trichophyton rubrum en ambos períodos y en el total de las muestras $(70,5 \%$ en el primero, $77,7 \%$ en el segundo y $73,9 \%$ del total de cultivos), y de las levaduras, la más frecuente fue Cándida parapsilosis en el primer período $(7,1 \%)$ y Cándida sp en el segundo período $(8,1 \%)$, y del total fue Cándida sp $(4,1 \%)$. Se determinó Cándida sp cuando no fue identificada específicamente la especie, por lo que podría incluir a otras especies de cándida.

En relación a los cultivos de HFND se obtuvieron en total 40 positivos $(1,2 \%)$, y destaca que la prevalencia de HFND aumentó significativamente entre los 2 perío$\operatorname{dos}(\mathrm{p}<0,0001)$ (Tabla 1). Las especies encontradas en total fueron: 23 Fusarium sp $(57,5 \%), 8$ Scopulariopsis sp (20\%), 7 Acremonium sp (17,5\%), 1 Aspergillus niger $(2,5 \%)$ y 1 Scedosporium sp $(2,5 \%)$. La prevalencia de cada una según el período se especifica en la Tabla 2. 
Tabla 1

Resultado de cultivos obtenidos en cada período

\begin{tabular}{|c|c|c|c|c|c|c|}
\hline \multirow[t]{2}{*}{ Períodos } & \multicolumn{2}{|c|}{ 2008-2009 } & \multicolumn{2}{|c|}{ 2016-2017 } & \multicolumn{2}{|c|}{ Ambos períodos } \\
\hline & $\mathbf{N}^{0}$ absoluto & Frec (\%) & $\mathrm{N}^{0}$ absoluto & Frec (\%) & $\mathbf{N}^{0}$ absoluto & Frec (\%) \\
\hline Cultivos total & 4985 & & 4594 & & 9579 & \\
\hline Cultivos hongos $(+)$ & 1831 & 36,7 & 1611 & 35 & 3442 & 35,9 \\
\hline Cultivos Pseudomona $(+)$ & 1 & 0,1 & 5 & 0,3 & 6 & 0,1 \\
\hline DERMATOFITOS & 1473 & 80,4 & 1398 & 86,8 & 2871 & $83,4 p=0,108$ \\
\hline T. rubrum & 1291 & 70,5 & 1256 & 77,7 & 2547 & 73,9 \\
\hline T. mentagrophytes & 178 & 9,7 & 133 & 8,2 & 311 & 9 \\
\hline E. flocosum & 3 & 0,2 & 3 & 0,2 & 6 & 0,2 \\
\hline Tricophyton sp & 1 & 0,1 & 3 & 0,2 & 4 & 0,1 \\
\hline Microsporum canis & 0 & 0 & 2 & 0,1 & 2 & 0,1 \\
\hline $\mathrm{T}$. Tonsurans & 0 & 0 & 1 & 0,1 & 1 & 0 \\
\hline LEVADURAS & 350 & 19,1 & 181 & 11,2 & 531 & $15,4 \mathrm{p}<0,001$ \\
\hline Candida albicans & 92 & 5 & 35 & 2,2 & 127 & 3,7 \\
\hline Candida sp & 20 & 1,1 & 131 & 8,1 & 151 & 4,4 \\
\hline Candida parapsilosis & 130 & 7,1 & 11 & 0,7 & 141 & 4,1 \\
\hline Candida guillermondi & 93 & 5,1 & 1 & 0,1 & 94 & 2,7 \\
\hline Candida tropicalis & 7 & 0,4 & 1 & 0,1 & 8 & 0,2 \\
\hline Candida lusitaniae & 5 & 0,3 & 2 & 0,1 & 7 & 0,2 \\
\hline Candida famata & 3 & 0,2 & 0 & 0 & 3 & 0,1 \\
\hline HFND & 8 & 0,4 & 32 & 2 & 40 & $1,2 \mathrm{p}<0,0001$ \\
\hline Fusarium sp & 5 & 0,3 & 18 & 1,1 & 23 & 0,7 \\
\hline Scopulariopsis sp & 2 & 0,1 & 6 & 0,4 & 8 & 0,2 \\
\hline Acremonium sp & 1 & 0,1 & 6 & 0,4 & 7 & 0,2 \\
\hline Aspergillus niger & 0 & 0 & 1 & 0,1 & 1 & 0 \\
\hline Scedosporium $\mathrm{sp}$ & 0 & 0 & 1 & 0,1 & 1 & 0 \\
\hline
\end{tabular}

Tabla 2

Hongos Filamentosos no Dermatofitos según períodos

\begin{tabular}{|c|c|c|c|}
\hline Períodos & 2008-2009 & 2016-2017 & Total \\
\hline & $\begin{array}{c}\mathrm{N}^{0} \text { absoluto } \\
(\%)\end{array}$ & $\begin{array}{c}\mathrm{N}^{0} \text { absoluto } \\
(\%)\end{array}$ & $\begin{array}{c}\mathrm{N}^{0} \text { absoluto } \\
(\%)\end{array}$ \\
\hline HFND total & $8(100)$ & $32(100)$ & 40 (100) \\
\hline Fusarium sp & $5(62,5)$ & $18(56,3)$ & $23(57,5)$ \\
\hline Scopulariopsis sp & $2(25)$ & $6(18,8)$ & $8(20,0)$ \\
\hline Acremonium sp & $1(12,5)$ & $6(18,8)$ & $7(17,5)$ \\
\hline Aspergillus niger & $0(0,0)$ & $1(3,1)$ & $1(2,5)$ \\
\hline Scedosporium sp & $0(0,0)$ & $1(3,1)$ & $1(2,5)$ \\
\hline Localización & & & $\mathrm{p}=0,59$ \\
\hline Manos & $1(12,5)$ & $2(6,3)$ & $3(7,5)$ \\
\hline Pies & $7(87,5)$ & $30(93,8)$ & $37(92,5)$ \\
\hline Sexo & & & $p=0,58$ \\
\hline Femenino & $5(62,5)$ & $16(50)$ & $21(52,5)$ \\
\hline Masculino & $3(37,5)$ & $16(50)$ & $19(47,5)$ \\
\hline
\end{tabular}

Del total, 37 (92,5\%) correspondían a muestras de pies y $3(7,5 \%)$ a muestras de manos, con similar proporción en ambos períodos. Con respecto al sexo, del total de muestras $52,5 \%$ fueron de sexo femenino, $47,5 \%$ mas- culino; en el primer período $62,5 \%$ sexo femenino y en el segundo $50 \%$ (Tabla 2). La edad promedio fue de 40 años $\pm 14,7$, con edades similares en ambos períodos. Además, no hubo diferencias significativas entre las especies de HFND y la localización $(p=0,86)$ ni el sexo $(p=0,57)$.

En los pacientes con cultivos para HFND, se analizaron antecedentes mórbidos, uso de inmunosupresores, tabaquismo y tratamientos previos, y no se encontraron asociaciones.

\section{Discusión}

Las onicomicosis causadas por HFND están emergiendo y se estima a nivel mundial que entre $1-17 \%$ de las onicomicosis son causadas por HFND; este rango es muy amplio, variando considerablemente según reportes de diferentes geografías (desde 4,89\% en Venezuela ${ }^{6}$ a 51,6\% en Tailandia ${ }^{13}$ ). La prevalencia de HFND de nuestro estudio fue de 1,2\%, la cual es menor en comparación con otros países ya mencionados. Estas variacio- 
nes se pueden deber a diferencias en factores locales de cada lugar geográfico, factores del huésped y de laboratorio, como las técnicas diagnósticas usadas, entre otros.

No hay consenso en los criterios diagnósticos de onicomicosis por HFND lo cual dificulta la interpretación entre los diferentes trabajos publicados. Los clásicos criterios de English ${ }^{18}$ (1976) pueden ser muy estrictos y han sido criticados por Gupta et al. ${ }^{19,20}$ La mayoría de los autores comparten los siguientes criterios para aceptar la patogenicidad de los HFND: 1) alteraciones ungueales clínicamente sugerentes de onicomicocis 2) examen micológico directo positivo 3) aislamiento exclusivo del hongo en un medio de cultivo 4) aislamiento confirmatorio repetido, al menos una vez, en las mismas condiciones. ${ }^{1,21}$ Shemer et al desarrollaron nuevos criterios, en los que proponen que si el paciente tiene un cultivo positivo para HFND, se debe citar nuevamente, tomar 3 muestras separadas de la uña afectada, y si se confirma en los 3 cultivos, se establece el diagnóstico. ${ }^{22}$

El medio de cultivo más usado es el agar Sabouraud, donde crecen la mayoría de los hongos, incluídos los HFND. Sin embargo, el uso de medios de cultivo con sustancias inhibidoras selectivas, como la cicloheximida, que impide el crecimiento de la mayoría de los mohos, puede subestimar la prevalencia de los HFND. Es por esto que es fundamental usar tubos con estas sustancias y otros no, tal como lo realizamos en nuestro laboratorio. ${ }^{23}$

La especie de HFND más frecuente en nuestro medio fue Fusarium sp con un 57,5\%, seguido por Scopulariopsis sp con un 20\% y Acremonium sp 17,5\%. Esto es concordante con otros estudios que muestran que Fusarium sp es la especie de los HFND más frecuente de Sudamérica. En los países europeos prevalece Scopulariopsis, Aspergillus, Acremonium y Fusarium. ${ }^{1}$

La presentación clínica de esta onicomicosis es generalmente inespecífica. En general, la mayoría involucra los dedos de los pies, al igual que en nuestro trabajo con 92,5\% uñas de pies. Se ha descrito que Fusarium, Aspergillus y Acremonium se manifiestan con una leuconiquia superficial, ${ }^{17}$ y que Scopulariopsis, Fusarium y Aspergillus suelen tener afectación proximal y paroniquia, aunque pueden ser indistinguibles de los dermatofitos. ${ }^{6}$

Al comparar la prevalencia de los dos períodos (2008 y 2009 versus 2016 y 2017), se vió que en tan solo 6 años ha habido un aumento significativo de cultivos positivos para HFND, aumentando de $8(0,4 \%)$ en el primero a
$32(2 \%)$ en el segundo. Estos datos concuerdan con lo expuesto en la literatura, donde se ha evidenciado un aumento de onicomicosis por HFND, tanto en pacientes inmunocomprometidos como en inmunocompetentes, en especial por Fusarium. ${ }^{7,22-24}$

Las razones particulares de este aumento las desconocemos, pero podemos descartar los factores relacionados al laboratorio (operadores, técnicas, medios de cultivo, lugar de trabajo y criterios diagnósticos) ya que no sufrieron cambios en ambos períodos.

Una limitación de nuestro trabajo es ser un estudio retrospectivo, por lo tanto, no se pudieron realizar cultivos en días diferentes ni re-examinar a los pacientes una vez que el cultivo resultara positivo, para poder tener la correlación clínico-microbiológica. Sin embargo, siempre se obtuvo al menos dos cultivos positivos para HFND y ausencia de crecimiento de otro patógeno. Por otra parte, no se obtuvieron todos los datos clínicos y epidemiológicos de los pacientes, pues las fichas clínicas a menudo estaban incompletas.

Hay que destacar que, de un total de 9.579 muestras, solo se obtuvieron $36 \%$ cultivos positivos, por lo que en la práctica clínica es fundamental la correlación clínicomicrobiológica y el juicio clínico. Se debe indagar en diagnósticos diferenciales y a su vez no limitar la decisión terapéutica exclusivamente a los resultados de los MD y cultivos. A pesar de que el cultivo de hongos tiene baja sensibilidad (cercano a 60\%), aún se considera como un gold estándar, pues otras técnicas (histolopatología y PCR) no están ampliamente disponibles en los centros.

Hay autores que han cuestionado la necesidad de cumplir todos los criterios de onicomicosis por HFND, pues casi el 50\% de las infecciones por HFND podrían subdiagnosticarse si se requiriera tanto el micológico directo como los cultivos positivos. Ellos consideran que el MD tiene una baja sensibilidad, por lo que, si se excluyen las muestras negativas, hay alta probabilidad de subdiagnosticar a los pacientes. Proponen evaluar aquellas muestras con al menos tres cultivos positivos, independiente del resultado del MD, para reducir la tasa de falsos negativos. ${ }^{5}$ La histopatología (onicohistotest) sería otra forma de complementar el diagnóstico e identificar el agente, evaluando una muestra de tejido ungueal en la cual se observa la presencia de hongos invadiendo la lámina ungueal. 


\section{Conclusión}

Presentamos un estudio transversal para describir la epidemiologia de onicomicosis en Santiago, Chile. Los HFND correponden al tercer patógeno causal de las onicomicosis. Este mostró que los HFND presentan prevalencia de $1,2 \%$ y predominan en las uñas de los pies. Las especies más frecuentes fueron Fusarium sp, Scopulariopsis sp y Acremonium sp. La prevalencia de HFND aumentó significativamente en un período de 6 años.

La importancia de este estudio radica en buscar de manera más activa los HFND con el fin de proteger a nuestros pacientes de infecciones que pueden tener resultados con alta morbi-mortalidad, especialmente en el caso de inmunosuprimidos.

\section{Agradecimientos}

Agreadecemos la colaboración de Héctor Fuenzalida Wong en la recolección de datos, y a Pilar León y Paulina Meza por ayudarnos en el trabajo en el laboratorio.

\section{REFERENCIAS}

1. Gupta AK, Drummond-Main C, Cooper EA, Brintnell W, Piraccini BM, Tosti A. Systematic review of nondermatophyte mold onychomycosis: Diagnosis, clinical types, epidemiology, and treatment. Journal of the American Academy of Dermatology. 2012 Mar;66(3):494-502

2. Gupta AK, Jain HC, Lynde CW, MacDonald P, Cooper EA, Summerbell RC. Prevalence and epidemiology of onychomycosis in patients visiting physicians' offices: A multicenter Canadian survey of 15,000 patients. Journal of the American Academy of Dermatology. 2000 Aug;43(2):244-8

3. Effendy I, Lecha M, Feuilhade de Chauvin M, Di Chiacchio N, Baran R. Epidemiology and clinical classification of onychomycosis. J Eur Acad Dermatol Venerol. 2005 Sep;19(s1):8-12

4. Ghannoum MA, Hajjeh RA, Scher R, Konnikov N, Gupta AK, Summerbell R, et al. A large-scale North American study of fungal isolates from nails: The frequency of onychomycosis, fungal distribution, and antifungal susceptibility patterns. Journal of the American Academy of Dermatology. 2000 Oct;43(4):641-8

5. Bombace F, Iovene MR, Galdiero M, Martora F, Nicoletti GF, D'Andrea M, et al. Non-dermatophytic onychomycosis diagnostic criteria: an unresolved question. Mycoses. 2016 Sep;59(9):558-65

6. Gavallera E, Asbati M. Onicomicosis por hongos filamentosos no dermatofitos. Dermatologia Venezolana 2006;44(1):4-10
7. Gupta AK, Ryder JE, Baran R, Summerbell RC. Non-dermatophyte onychomycosis. Dermatologic Clinics. 2003 Apr;21(2):257-68

8. Galletti J, Negri M, Grassi FL, Kioshima-Cotica ÉS, Svidzinski TIE. Fusarium spp. is able to grow and invade healthy human nails as a single source of nutrients. Eur J Clin Microbiol Infect Dis. 2015 Sep;34(9):1767-72

9. Tovar LJM, Gayosso PM, Berruecos RAR, Silva I, Hernández FH, Martínez RL. Frecuencia de onicomicosis por hongos filamentosos no dermatofitos en un hospital de tercer nivel. Dermatol Rev Mex 2013;57:235-239

10. Escobar ML, Carmona-Fonseca J. Onicomicosis por hongos ambientales no dermatofíticos. Revista Iberoamericana Micología. 2003;20:6-10

11. Velez A, Linares J, Fenandez-Roldan JC, Casal M. Study of onychomycosis in Córdoba, Spain: Prevailing fungi and pattern of infection. Mycopathologia 1997;137:1-8

12. Ramani R, Srinivas GR, Ramani A, Kumari TGR, Shivananda PG. Molds in Onychomycosis. Int J Dermatol. 1993 Dec;32(12):877-8

13. Ungpakorn R, Lohaprathan S, Reangchainam S. Prevalence of foot diseases in outpatients attending the Institute of Dermatology, Bangkok, Thailand. Clin Exp Dermatol. 2004 Jan;29(1):87-90

14. Cruz Ch R, Ponce E E, Calderón R L, Delgado V N, Vieille O P, Piontelli L E. Micosis superficiales en la ciudad de Valparaíso, Chile: Período 2007-2009. Rev chil infectol. 2011 Oct;28(5):40-409

15. Alvarez MI, González LÁ, Castro LÁ. Onychomycosis in Cali, Colombia. Mycopathologia. 2004 Jul;158(2):181-6

16. Al-Hatmi AMS, Bonifaz A, Ranque S, Sybren de Hoog G, Verweij $\mathrm{PE}$, Meis JF. Current antifungal treatment of fusariosis. International Journal of Antimicrobial Agents. 2018 Mar;51(3):326-32

17. Varon AG, Nouer SA, Barreiros G, Trope BM, Magalhães F, Akiti T, et al. Superficial skin lesions positive for Fusarium are associated with subsequent development of invasive fusariosis. Journal of Infection. 2014 Jan;68(1):85-9

18. English MP. Nails and Fungi. BrJ Dermatol. 1976 Jun;94(6):697-701

19. Gupta AK, Cooper EA, MacDonald P, Summerbell RC. Utility of Inoculum Counting (Walshe and English Criteria) in Clinical Diagnosis of Onychomycosis Caused by Nondermatophytic Filamentous Fungi. Journal of Clinical Microbiology. 2001 Jun $1 ; 39(6): 2115-21$

20. Gupta AK, Ryder JE, Summerbell RC. The diagnosis of nondermatophyte mold onychomycosis. International Journal of Dermatology. 2003;42:272-3

21. Ranawaka RR, Nagahawatte A, Gunasekara TA. Fusarium onychomycosis: prevalence, clinical presentations, response to itraconazole and terbinafine pulse therapy, and 1-year follow-up in nine cases. Int J Dermatol. 2015 Nov;54(11):1275-82

22. Shemer A, Davidovici B, Grunwald MH, Trau H, Amichai B. New criteria for the laboratory diagnosis of nondermatophyte moulds in onychomycosis. British Journal of Dermatology. 2009 Jan;160(1):37-9

23. Lipner SR, Scher RK. Onychomycosis. Journal of the American Academy of Dermatology. 2019 Apr;80(4):835-51 